\title{
Poétique du corps enfermé. Répression et résistance des prisonnières politiques argentines (1976-1983)
}

Parler de «corps enfermé » paraît toujours être une sorte d'oxymore ou d'antithèse. Il semble en effet tellement étrange de penser que des êtres humains peuvent disposer de la liberté d'autrui sous divers prétextes. Or, il s'avère presque «normal » de considérer que certaines personnes doivent être enfermées et que d'autres doivent légiférer à ce propos. Les raisons de l'enfermement sont malheureusement tellement variées que certaines limites peuvent être rapidement franchies. C'est le cas de l'emprisonnement politique qui fait l'objet de notre article. Plus précisément, l'enfermement féminin sous la dernière dictature argentine, prenant comme point d'appui l'expérience des prisonnières politiques dans les établissements pénitentiaires de San Martín et Villa Devoto pendant la période qui va de 1976 à 1983.

Le croissant intérêt pour la récupération de la mémoire en Argentine a donné lieu à la publication de nombreux témoignages et, dans une moindre mesure, d'anthologies composées d'écrits carcéraux en général. C'est la raison pour laquelle nous nous appuierons principalement sur le livre collectif des anciennes prisonnières politiques, publié en 2006: Nosotras, presas políticas. Ce livre sera le point d'ancrage de notre réflexion qui se centrera sur les conditions d'enfermement dans deux des prisons les plus importantes du pays : San Martín et Villa Devoto. Ensuite, nous aborderons le sens de la féminité en prison sous un régime autoritaire et la façon dont les détenues ont trouvé le moyen de résister, grâce, notamment, à leur condition de femme.

\section{San Martín et Villa Devoto: centres de détention et de répression}

Avant d'analyser la résistance des femmes en prison, il convient de rappeler les conditions d'enfermement auxquelles elles étaient soumises. Tout d'abord, la répartition des femmes n'était pas aléatoire et correspondait à l'application de décrets visant à regrouper les prisonniers politiques (hommes et femmes) dans des prisons prévues à cet effet, c'est-à-dire aptes à recevoir des détenus politiques. Plus précisément, le décret 1209 du 6 juillet 1976 prévoyait que les individus dits « délinquants subversifs » soient concentrés dans des centres de détention de haute sécurité, dépendants du Service Pénitentiaire Fédéral à partir de fin 1976. C'est la raison pour laquelle des transferts massifs s'effectuèrent depuis les prisons de l'intérieur du pays jusqu'aux prisons habilitées à cet effet : Rawson Unité 6 (province de Chubut), Resistencia U7 (province du Chaco), Santa Rosa U4 (province de La Pampa), Villa Devoto U2 (province de Buenos Aires), La Plata U9 (province de Buenos Aires), Sierra Chica U2 (province de Buenos Aires), Coronda U1 (province de Santa Fe).

La modification de la carte de "distribution" des prisonniers politiques impliquait également la restructuration de certaines unités carcérales. Certaines d'entre elles ont dû subir des modifications afin d'appliquer le traitement prévu pour les détenus. La suppression des visites de contact, par exemple, a engendré la construction de parloirs vitrés dans les prisons qui n'en étaient pas pourvues. De même, les sanctions devaient se purger dans des cachots, qui durent être bâtis dans certains établissements. Enfin, il convient de mentionner l'édification d'une nouvelle unité carcérale qui est 
entrée en fonctionnement à partir de 1979 : Caseros, Unité Pénitentiaire 1, province de Buenos Aires ${ }^{1}$.

Évidemment, les femmes et les hommes étaient répartis dans différents centres de détention et les femmes furent finalement concentrées dans la prison de Villa Devoto de la province de Buenos Aires. Cependant, il n'est pas négligeable de mentionner que l'Unité Pénitentiaire $N^{\circ} 1$ (UP1) de San Martín de la province de Córdoba fonctionna pendant un certain temps comme l'une des prisons les plus rudes, tant pour les hommes (répartis dans les anciens bâtiments) que pour les femmes (réparties dans la partie neuve). D'ailleurs, il est possible d'affirmer que la UP1 de San Martín est un exemple emblématique qui illustre la relation ambigüe entre la répression légale et illégale. En effet, tous les témoignages s'accordent sur le fait que cette prison était particulièrement féroce et ce, en dépit du caractère légal de la situation pénale du détenu. Pour cause, une différence notoire entre les Centres Clandestins de Détention (CCD) et les prisons résidait dans le statut juridique de l'arrestation. Dans les CCD, les personnes étaient séquestrées et considérées, aux yeux de la société et de la justice, comme «disparues »; par conséquent, la torture et la durée de l'enfermement étaient sans limites. En revanche, la légalisation du détenu provoquait souvent chez les individus une sorte de soulagement, malgré les nombreux abus tout de même commis dans la légalité, comme le fut l'assassinat de 31 personnes (dont 6 femmes) commis dans l'enceinte de la prison de San Martín. En d'autres termes, l'incertitude quant à la survie était constante.

La prison de San Martín a reçu jusqu'à cent-trente détenues en même temps, lesquelles étaient logées dans le pavillon 14 où elles occupaient les deux premiers étages tandis que le troisième était réservé aux cachots ou cellules de châtiments. L'absence totale de communication a commencé à partir du coup d'État du 24 mars $1976^{2}$ dans la mesure où cette date a marqué un changement drastique dans les conditions d'enfermement à cause de l'intervention des forces militaires, comme le rappelle une ancienne prisonnière :

Jusqu'au coup d'État du 24 mars 1976, nous avions des visites de nos familles, il était permis de lire, de travailler, d'étudier, les mères pouvaient être avec leurs enfants jusqu'à leur trois ans [...]. Quand les militaires sont entrés, après le 24 mars, ils ont tout détruit. En une semaine, ils ont emporté les enfants, le papier, les crayons, les livres, les éléments d'hygiène, même le contact avec nos camarades de détention. ${ }^{3}$

\footnotetext{
${ }^{1}$ Il est aussi très important de mentionner la partie clandestine de l'enfermement. En effet, il est aujourd'hui impossible de parler de la dictature argentine sans faire référence aux 30.000 disparus et aux Centres Clandestins de Détention (CCD) qui ont atteint le nombre de 600. Si l'on ne s'attarde pas ici sur cet aspect, c'est parce que peu de CCD furent à la fois des centre de détention et de création alors que la plupart des prisons « légales » le furent. Néanmoins, rappelons que les relations entre le système illégal et le système légal étaient on ne peut plus étroites dans la mesure où certains prisonniers arrivaient en prisons avec des marques de torture, preuve de leur passage préalable par un CCD. In Calveiro 2006 : 137.

${ }^{2}$ L'absence de communication était permanente sauf à Noël où une visite a été autorisée avec pas plus de trois membres de la famille.

${ }^{3}$ Galará 2006 : 26-27. Nous traduisons. « Hasta antes del golpe de Estado del 24 de marzo de 1976 teníamos visitas de nuestros familiares, estaba permitido leer, trabajar, estudiar, las mamás podían quedarse con sus hijos hasta tres años [...]. Cuando irrumpieron los militares, después del 24 de marzo, entraron destrozando todo. En el lapso de una semana se llevaron los niños, los papeles, los lápices, los libros, los elementos de higiene, hasta el contacto con nuestras compañeras de detención. »
} 
Comme nous pouvons le constater, presque tout a fini par être interdit, sauf la réception en quantité extrêmement contrôlée et limitée de colis en provenance des familles, qui approvisionnaient les détenues en matériel d'hygiène (savon, dentifrice, serviettes hygiéniques, coton, etc.). Les autorités pénitentiaires mirent tout en œuvre afin d'empêcher la communication entre les prisonnières et avec l'extérieur. Pour ce faire, la correspondance et les visites furent interdites, et les femmes étaient réparties dans des cellules individuelles de 2 mètres 10 sur 1 mètre 40, illuminées 24 heures sur 24, desquelles elles ne pouvaient sortir que deux heures par jour (sous réserve de ne pas être sanctionnée pour quelque motif que ce soit). Quant aux sanctions, elles étaient permanentes et totalement arbitraires, comme le démontrent les archives du Service Pénitentiaire Provincial dans lesquelles sont mentionnés certains motifs de châtiments dans les dossiers personnels des prisonnières : «Lui sont appliqués 15 jours sous clefs dans sa cellule pour s'être moquée des employées du pavillon 14. ${ }^{4}$.

La UP1 était malheureusement célèbre parmi les prisonniers pour sa dureté et le maintien des détenus dans un état d'alerte permanent. Nous avons déjà mentionné le statut semi-légal de la prison qui, malgré la légalisation de la détention des prévenus, a été le théâtre de nombreuses fusillades. Il convient également de rappeler que les transferts de prisonniers étaient extrêmement fréquents. Ceux-ci avaient pour but de semer la terreur au sein de la prison car l'individu transféré se voyait à nouveau dépourvu de protection légale et juridique. En quelques sortes, il redisparaissait et subissait, par la même occasion, les sévices corporels et psychologiques propres aux $\mathrm{CCD}^{5}$. Des documents officiels attestent de la complicité du système pénitentiaire légal avec le système clandestin, comme le prouve le document suivant dans lequel l'Armée ordonne le transfert d'une détenue, transfert autorisé par le tampon de l'administration pénitentiaire : «À partir de la réception de ce courrier, monsieur le Directeur aura l'obligeance d'ordonner la remise de la détenue dont le nom apparaît ci-dessous, dans le but d'être interrogée. ${ }^{6}$. Il est clair et avéré que le langage utilisé par les autorités pénitentiaires et militaires était parsemé d'euphémismes. Cet extrait en est un exemple car l'interrogatoire qui y est mentionné est une façon atténuée de nommer l'obtention d'informations sous la torture menée dans un CCD où la personne est conduite ${ }^{7}$.

La UP1 de San Martín ne faisait pas partie des prisons mentionnées dans le décret 1209 du 6 juillet 1976, qui établissait que la nouvelle catégorie de détenus « délinquants subversifs » soit concentrée dans des prisons de haute sécurité du Service Pénitentiaire Fédéral. Pour cette raison, sauf quelques prisonnières, presque toutes les femmes avaient été transférées à la prison de Villa Devoto de Buenos Aires fin 1977. Le dernier transfert à Devoto s'effectue au début de l'année 1981 et il ne reste alors qu'une seule femme à San Martín qui sera transférée à la prison du Buen Pastor de Córdoba en avril 1981.

\footnotetext{
${ }^{4}$ Extrait de la fiche personnelle d'Ana Mohaded, in Comisión Provincial de la Memoria 2009 : 37.

5 N'oublions pas cependant que la torture était aussi pratiquée dans les prisons légales. Toutefois, la différence réside dans sa durée et sa cruauté, qui ont atteint des niveaux absolument inimaginables dans les CCD.

${ }^{6}$ Comisión Provincial de la Memoria 2009 : 44. Ver copia del original en el anexo I.2, p. 398.

${ }^{7}$ Il est important de signaler que les transferts pouvaient parfois s'effectuer dans le but de retenir un prisonnier en otage et annuler toute tentative de résistance dans ou en dehors de la prison (de la part des organisations politiques opposées à la dictature). Ce fut le cas lors de la visite de la Croix Rouge Internationale en avril 1978: 15 prisonniers politiques (3 femmes et 12 hommes) furent transférés au CCD Campo de la Ribera de Córdoba, condamnés à mort si certains détenus dénonçaient les violations des droits de l'homme.
} 
À cause de la répression extrême soufferte à la UP1 de San Martín, l'arrivée à Devoto s'est parfois vécue comme un soulagement, comme le prouve le témoignage d'Edelveis Gallegos:

J'ai arrêté de respirer jusqu'à ce qu'ils me fassent entrer dans un bureau. Là, ils m'ont enlevé le bandeau des yeux et j'ai vu un homme derrière un bureau. Il a commencé à noter mes coordonnées, alors j'ai osé et je lui ai demandé où j'étais et il m'a répondu «Comment ça, vous ne le savez pas?». Et il a continué «dans la prison la plus grande d'Amérique du Sud ». «Où », ai-je demandé et il m'a répondu «à Villa Devoto », je lui ai demandé s'il y avait des visites, si je pouvais envoyer et recevoir du courrier, si nous avions des sorties et il me répondait oui à tout. Cela faisait plus de 15 heures que je n'avais pas bu ni été aux toilettes, mais ces réponses m'ont fait tellement plaisir que je me serais crue dans un hôtel des Caraïbes. ${ }^{8}$

Comparer Villa Devoto à un "hôtel des Caraïbes" révèle le contraste avec les conditions d'enfermement de San Martín. Villa Devoto, également connue comme la "prison vitrine", fut la principale unité de détention de femmes pendant la dictature et est allée jusqu'à recevoir environ deux mille détenues entre 1975 et 1983, année où elle s'est vidée pour loger des prisonniers politiques.

Devoto était une prison légale, mais en dépit du fait que le degré de répression n'y était pas aussi élevé qu'à San Martín, il est tout de même nécessaire de préciser qu'une politique autoritaire s'appliquait quotidiennement. Aucun assassinat n'y a été perpétré, cependant, de nombreux transferts de prisonnières vers des CCD ont été effectués afin de les interroger et torturer illégalement, et de les retenir en otages durant des épisodes tels que la visite d'organismes des droits de l'homme, par exemple (Croix Rouge Internationale, Commission Interaméricaine des Droits de 1'Homme, etc.). De plus, des interrogatoires ont été menés au sein de la prison afin de connaitre l'organisation interne des détenues: «Et un jour ils l'ont ramenée. Ils l'avaient interrogée et torturée tout en la frappant et en lui appliquant la gégène pendant qu'ils lui demandaient: 'Comment vous fonctionnez à Devoto ?' 'Qui parle de politique?' 'Qui sont celles qui commandent ?’ »'.

Si la dégradation des conditions d'enfermement fut soudaine à la UP1, en revanche, elle s'effectua par étape à Devoto. Un premier changement se produisit au milieu de l'année 1975 par l'application du décret 2023/74 qui restreignit l'entrée de livres, journaux, les sorties internes et les visites. À ce moment-là, les femmes étaient concentrées dans le pavillon 49 dans une cellule de 20 mètres sur 9 où elles pouvaient être avec leurs enfants ${ }^{10}$; ensuite, elles furent réparties dans huit pavillons. Le mois de

\footnotetext{
${ }^{8}$ VV.AA 2006 : 149. Nous traduisons. « Me quedé casi sin respirar, hasta que me hicieron pasar a una oficina. Allí me sacaron las vendas de los ojos y vi a un hombre detrás de un escritorio. Este comenzó a tomarme los datos, entonces me animé y le pregunté dónde estaba y me contestó “¿cómo no lo sabe?”. Y siguió “en la cárcel más grande de Sudamérica” ¿dónde? pregunté y me contestó "en Villa Devoto", le pregunté si aquí había visitas, si podía enviar y recibir cartas, si teníamos recreo y a todo me contestaba que sí. Había pasado más de 15 horas sin tomar nada ni ir al baño, pero estas respuestas me dieron tanta alegría como si hubiera llegado a un hotel del Caribe. »

${ }^{9}$ VV.AA 2006 : 270. Nous traduisons. «Y, así, un día la trajeron de vuelta. La habían interrogado y torturado con picana eléctrica y golpes mientras le preguntaban: ‘¿Cómo funcionan en Devoto?' ¿Quién habla de política?' ‘Quiénes son las que mandan?’ ».

${ }^{10}$ À cette période, il y avait douze enfants pour soixante-sept détenues. VV.AA 2006 : 47.
} 
novembre 1975 a marqué une nouvelle étape puisque la prison a commencé à dépendre de l'Armée qui, en décembre, prohiba la communication avec l'extérieur, les sorties internes, les visites, la correspondance, les journaux et la réception de colis jusqu'à janvier $1976^{11}$.

Le coup d'État de 1976 enclencha une nouvelle étape dans la mesure où des détenues commencèrent à arriver avec des marques visibles de torture et à partir de septembre, des arrivées massives de femmes en provenance des provinces de Córdoba, Tucumán, Santa Fe, Chaco, Mendoza et Santiago del Estero s'effectuèrent, élevant le nombre de détenues à mille deux-cents en fin d'année. Elles étaient quatre dans des cellules de 2 mètres 50 sur 3 mètres 50, enfermées dix-sept heures par jour avec deux sorties internes (couloirs) et une sortie externe d'une heure (cour) ${ }^{12}$. Elles avaient l'obligation de se doucher quotidiennement même si l'eau était glaciale en hiver. Cependant, à mesure que les mois avançaient, quelques améliorations se sont faites sentir, comme la possibilité de correspondre à nouveau avec un membre certifié de la famille, ou encore l'autorisation de recevoir des visites, des colis et d'obtenir du matériel pour écrire (crayons, cahiers), tout cela en quantité extrêmement limitée.

L'année 1977 fut marquée par la construction des parloirs vitrés dont Villa Devoto n'était pas encore pourvue, ce qui affecta grandement le moral des détenues, en particulier, celui des mères. De plus, le recours aux châtiments (dans des cachots) et aux fouilles (corporelles et des cellules) s'intensifia. Il convient également de mentionner la mise en application d'un projet pour le moins pervers, visant à la division et destruction de la «subversion»: la classification des détenues. Ceci consistait en la création de trois groupes de prisonnières, qui étaient classées selon leur «dangerosité »: les « irrécupérables» constituaient le groupe 1 (G1) tandis que celles qui étaient «possiblement récupérables » constituaient le G2 et les «récupérables » le G3 ${ }^{13}$. Ce schéma pervers, nommé ironiquement «plan de la carotte ou du bâton » par les détenues $^{14}$, tendait à instaurer la méfiance entre les femmes pour ainsi inciter à la repentance et à la remise d'informations en échange de «récompenses » : davantage de nourriture, de visites, de sorties, de lectures, etc.

Ce ne fut qu'en 1981 que de véritables changements commencèrent à s'appliquer. Les détenues purent à nouveau recevoir des visites de contact, faire des travaux manuels et des activités physiques, obtenir des journaux, correspondre avec des membres de leur famille enfermés dans d'autres prisons ${ }^{15}$, etc.

\section{Faire face: de l'abus au "coup de culotte"}

Malgré la dureté des conditions d'enfermement décrites précédemment, il est important de signaler que les femmes mirent absolument tout en œuvre afin de construire une véritable politique de résistance, un réseau qui a permis que la majorité des femmes se sentent soutenues.

\footnotetext{
${ }^{11}$ VV.AA $2006: 66-68$.

12 VV.AA $2006: 108-109$.

${ }^{13}$ Garaño et Pertot $2007: 27$.

${ }^{14}$ VV.AA 2006 : 216.

${ }^{15}$ Il faut savoir que jusque-là, la correspondance n'était admise qu'avec des membres certifiés et libres de la famille. À partir de fin 1979 et début des années 80, les prisonnières purent écrire à leurs maris, frères, etc. enfermés dans d'autres établissements pénitentiaires du pays. En 1981, cette pratique s'est répandue.
} 
$\mathrm{Au}$ vu des conditions extrêmes souffertes à San Martín, nous pourrions croire que les actes de résistance furent isolés. Cependant, les détenues devinrent fameuses grâce au courage qu'elles démontrèrent lors des fouilles corporelles puisqu'elles décidèrent de refuser, en groupe, de baisser leur culotte. Si l'on situe cet épisode, connu comme «le coup de culotte » dans son contexte, cela prend tout son sens puisque les prisonnières s'exposaient, en pleine dictature (1977), à un châtiment certain qui pouvait aller de l'isolement à la mort ${ }^{16}$. Il faut savoir que ce «coup de culotte » fut un épisode clefs dans le processus de résistance car c'était une façon de se réapproprier d'un corps qui avait été de nombreuses fois bafoué et maltraité. N'oublions pas que la persécution envers les femmes avait un caractère sexuel et systématique. D'une certaine façon, le corps de la militante s'est transformé, pendant la dictature, en « réceptacle » de la haine militaire. Si le viol et le vol de bébés sont des crimes qui se sont commis en Argentine et dans plusieurs parties du monde, c'est qu'il existe des codes imperceptibles ou hautement perceptibles -selon le point de vue adopté- qui considèrent que ces situations conflictuelles octroient la «permission »de franchir certaines frontières. Estimer que la femme, dans des situations extrêmes perd ses "frontières", entendues comme les contours de son corps, est une appréciation malheureusement automatique, comme si son corps était la matérialisation de l'ennemi, la citadelle à pénétrer à tout prix pour y déverser des flots de haine et s'assurer qu'ils y resteront pour toujours. Les crimes de "profanation" comme les nomme l'anthropologue Ludmila da Silva Catela ont tendance à être classés parmi les tortures "courantes" pendant une situation conflictuelle, ce qui incite les victimes à les taire ${ }^{17}$. Selon elle, le tabou qui concerne le viol est la conséquence directe de la difficulté, pour l'auditoire, à écouter des témoignages semblables, d'une part. D'autre part, elle rappelle que ce crime n'est pas considéré d'un point de vue politique, ce qui constitue un obstacle à la reconstruction personnelle ${ }^{18}$.

Partant de ce principe, il est plus aisé de comprendre l'origine d'un mouvement de résistance en prison comme le fut le « coup de culotte ». Par cet intermédiaire, les femmes ne s'opposaient pas seulement aux fouilles dénigrantes, mais elles s'érigeaient comme des êtres à part entière aux yeux des autorités pénitentiaires. En d'autres termes, dans un contexte qui tendait à l'annulation de l'humanité et de la dignité humaine, les femmes se sont proclamées comme des êtres pensants et agissants, dotées d'un surprenant pouvoir de décision et d'opposition. La ténacité et la volonté des femmes finirent par représenter, au sein de la prison, une autorité impossible à ignorer et dominer puisque grâce à cette rébellion et en dépit de recevoir une sanction collective, les femmes ne durent plus baisser leur culotte pendant la fouille corporelle. Dans ce sens, parler de «coup de culotte », équivaut presque à parler de micro-révolution puisque les femmes ont, d'une certaine manière, récupéré une partie de leur corps qui abandonna son statut public (fouillé, exposé, torturé) pour retrouver son statut privé et intime.

L'existence d'un épisode comme le "coup de culotte" invite à réfléchir aux conditions qui l'ont permis. Or, il s'avère très vite que tout empêchait sa réalisation: la répression quotidienne, la répartition en cellules individuelles et l'absence presque

\footnotetext{
${ }^{16}$ Mohaded 2010.

${ }^{17}$ Da Silva Catela in Comisión y Archivo Provincial de la Memoria mai 2010 : 2.

${ }^{18}$ Aujourd'hui, Claudio Orosz, avocat plaignant dans le procès pour crimes contre l'humanité Megacausa La Perla de Córdoba, a déclaré que les délits sexuels allaient faire partie de la cause et être considérés comme crimes contre l'humanité. In Platía 19 août 2013.
} 
totale de communication rendaient absolument ardue la préparation d'un quelconque acte collectif de résistance. Cependant, il a existé et a émané des femmes qui ont su développer, en prison, un esprit de résistance indéniable, à tel point que le Chef de la Sécurité de l'époque déclara:

«Vous n'êtes pas une fille, vous êtes un être subversif et vous le resterez toujours. ». Je ne me souviens pas si ce fut cette fois-là ou une autre qu'il me dit: «Je préfèrerais qu'ils m'envoient tous les chefs guérilléros que de traiter avec ces folles. Les femmes sont pires, quand elles croient en quelque chose elles le sentent dans leurs entrailles. Les mecs sont plus raisonnables. ${ }^{19}$

Si des différences notoires s'observent entre la résistance masculine et féminine pendant la même période, ceci s'explique en grande partie par le fait que les femmes ont paru trouver davantage de ressources pour supporter certaines situations. On remarque notamment qu'aucun suicide féminin n'a été enregistré en prison. Or, du côté des hommes, huit suicides ont été signalés ${ }^{20}$. Cette information est intéressante et révélatrice du réseau solidaire qui s'est tissé entre les détenues. Cela s'explique également par le fait que les femmes finirent par être toutes concentrées à Villa Devoto, contrairement aux hommes qui étaient répartis entre quatre grandes prisons fédérales (sans compter les prisons provinciales) et constamment transférés d'un centre de détention à un autre.

Il convient également de mentionner que les femmes furent perpétuellement animées de la volonté de partager. D'ailleurs, presque tout était pensé de manière collective: de la notion de survie à la maternité et la création artistique. Par exemple, les enfants -nés en captivité ou à l'extérieur- étaient les enfants de toutes. En revanche, il n'est fait mention dans aucun témoignage de la notion communautaire de la paternité. Nous pourrions même rajouter que la négation de la maternité, dans les CCD et dans les prisons légales faisait partie intégrante du châtiment systématique dont furent victimes les détenues alors que la négation de la paternité peut avoir été perçue davantage comme la conséquence "naturelle » de l'enfermement, dans une société qui a tendance à octroyer le rôle éducatif principal à la mère. À ce propos, il est important de rappeler que pas moins de cinq cents bébés furent volés dans les CCD, dans le but de les «sauver», au sens messianique du terme, de leurs parents «subversifs ». Dans ce sens, la négation de la maternité ou devrions-nous dire, l'appropriation de l'objet de la maternité fut donc une stratégie pour détruire les femmes dans leur essence. De cette façon, les militaires remettaient en question non seulement l'engagement politique des détenues mais aussi leur capacité à éduquer un enfant selon le modèle de société défendu par le pouvoir dictatorial, dont les valeurs principales tournaient autour de la patrie, la famille et l'église ${ }^{21}$. Comme le rappelle José García Romeu, l'Armée prétendait sauver le peuple entier, par conséquent, le vol de bébés s'inscrit totalement dans le projet militaire : "Comparé dès lors à une grande famille dont l'Armée aurait la charge patriarcale, le peuple devient une vue de l'esprit, une essence privée de son corps social et soumise aux militaires."22.

\footnotetext{
${ }^{19}$ VV.AA $2006: 210$. Nous traduisons.

${ }^{20}$ Il faut tout de même signaler que les circonstances de certains suicides n'ont toujours pas été éclaircies. Il est donc possible que parmi ces huit décès, quelques uns aient été des assassinats camouflés par les autorités pénitentiaires. Garaño et Pertot $2007: 317$. 21

${ }^{22}$ Ibid., p. 32. "Comparado desde entonces a una gran familia de la cual el Ejército tendría el cargo patriarcal, el pueblo se convierte en espejismo, una esencia privada de su cuerpo social y sumisa a los militares.”. La traducción es mía.
} 
Pour en revenir à la question du partage, l'ouverture et l'analyse des cahiers de la prison, que les détenues pouvaient acheter ou recevoir dans les colis en quantité limité, mettent en évidence l'étendue de cette notion. En effet, il s'avère que les femmes avaient l'habitude de partager les chansons et poèmes qu'elles y recopiaient. Cela concerne également les compositions inédites dont certaines figurent dans plusieurs cahiers à la fois. Cette pratique n'était pas si courante chez les hommes qui avaient tendance à être plus pudiques quant au contenu de leur cahier et à leurs sentiments en général. D'ailleurs, ceci peut s'observer dans certains livres de témoignage publiés en démocratie. Le livre des prisonnières politiques, Nosotras presas políticas, par exemple, en plus d'inclure des poèmes, des lettres et des dessins à la fin de chaque chapitre, est accompagné d'un CD sur lequel sont copiées cinq cents lettres écrites aux familles entre 1975 et 1983. Nous pouvons aussi citer le livre testimonial de Delia Galará qui consacre un chapitre à la correspondance et y recopie quelques unes de ses lettres. Enfin, l'écrivaine Alicia Kozameh a permis la publication digitale de l'intégralité de ses deux cahiers de Devoto ${ }^{23}$. En revanche, les hommes qui ont publié des ouvrages testimoniaux ont généralement intégré les œuvres artistiques (poèmes, dessins, chansons) sans inclure les lettres. Il en existe cependant quelques unes publiées dans des ouvrages de femmes $^{24}$. En résumé, l'initiative de s'ouvrir à l'autre a été un facteur décisif dans le processus de résistance individuel et collectif des femmes enfermées.

Résister face à la violence physique et morale est passé par la revendication du corps comme propriété de chacune au travers d'actions comme le «coup de culotte ». Mais il est aussi important de mentionner les actes quotidiens qui ont participé à la récupération du corps comme entité privée et éminemment féminine. De cette façon, le moindre élément pouvait servir au maquillage, par exemple, comme le rappellent Gladys Regalado et Sara Waitman: "Gladys: Certaines se faisaient une coiffe avec du papier hygiénique ou nous nous maquillions les yeux avec des bouts de charbon. Sara: Et les couvercles des boîtes de lait [marque] Nido étaient les miroirs." ${ }^{25}$. Malgré la fervente volonté d'aller à l'encontre de la politique de destruction physique et morale des autorités pénitentiaires, les détenues eurent du mal à réprimer certaines réactions naturelles que le corps tend à imposer en période de survie, comme l'aménorrhée, extrêmement fréquente en prison, selon Ana Mohaded :

La situation dans laquelle nous nous trouvions nous violentait, car du fait de ne pas avoir de serviettes hygiéniques, ni d'intimité dans la toilette, nous devions découper des serviettes pour fabriquer des petits mouchoirs que nous devions laisser dans les cellules jusqu'à ce qu'on nous ouvre la porte. Et s'il y avait une fouille c'était désagréable. Ils nous criaient «dégoûtantes». Face à cela, nous avons presque toutes cessé d'avoir nos règles. Ce fut conne une neutralisation de notre féminité. ${ }^{26}$

\footnotetext{
${ }^{23}$ Disponibles dans les Archives Virtuelles du Centre de Recherches Latino-américaines de l'Université de Poitiers, France. [URL : http://www.mshs.univ-poitiers.fr/crla/contenidos/Kozameh/].

24 Il s'agit généralement de lettres reçues par les auteures, écrites par leur compagnon et inclues postérieurement à la publication.

${ }^{25}$ Regalado et Waitman in Comisión y Archivo Provincial de la Memoria 2010 : 11. Nous traduisons. « Gladys : Algunas se hacían la toca con el papel higiénico o nos pintábamos los ojos con pastillas de carbón. Sara: Y las tapas de leche Nido eran los espejitos. »

26 Mohaded in Comisión y Archivo Provincial de la Memoria 2010 : 11. Nous traduisons. « Nos violentaba la situación en que nos sentíamos, porque al no tener pañitos, ni intimidad en la higiene, teníamos que cortar las toallas armar unos pañitos que debíamos dejar en la celda y estar con ellos hasta
} 
La «neutralisation » de la féminité, comme la nomme Ana Mohaded, cherchait justement à désorienter les détenues qui, finalement, ne pouvaient expérimenter ni le fait d'être femme ni celui d'être mère. En plus de ne pas avoir leurs règles et de se voir arrachés leurs enfants, les femmes durent utiliser un uniforme carcéral et supporter l'enfermement qui leur provoquait une dépigmentation faute de soleil, à laquelle elles tentaient de remédier en se rougissant les joues et les lèvres à l'aide de l'emballage rouge du paquet d'herbe à mate ${ }^{27}$ de marque Taragüí, ou de betterave pour ne pas paraître trop blanches lors des visites familiales. Parmi les procédés de féminisation, nous pouvons également mentionner la création du salon de coiffure «Yerutí » à Devoto -créé en 1977 et très vite fermé par les autorités pénitentiaires-, où l'on coupait les cheveux avec des lames de rasoir, faisait des soins du visage avec de la mie de pain mouillée à la place du coton, et s'épilait avec de la cire fabriquée à base de sucre et de citron $^{28}$. Les femmes avaient aussi l'habitude d'utiliser la suie de la bouilloire pour se maquiller les yeux, de la pommade anti-hémorroïdes pour faire briller les lèvres, etc. Sans oublier les diverses activités effectuées dans la clandestinité de la cellule pour occuper le temps et mettre de la vie et de la couleur au quotidien: artisanat en tout genre, crochet et tricot à partir de fil de serviette de toilette, dessin avec du cirage, sculpture d'os de viande de bœuf, etc.

Si la résistance s'est exprimée sous de nombreuses formes, il en est une qui mérite de plus amples explications : la poésie. Ce genre littéraire n'a pas été le propre des femmes, loin de là, puisque les hommes se sont également révélés être de grands poètes face à l'enfermement. Mais il est intéressant de différencier les deux écritures dans la mesure où le genre (masculin-féminin) influença pour beaucoup la production poétique carcérale. De cette façon, il est possible d'observer une différence notoire entre les deux poésies, qui tient dans la manière de résister à l'absence ${ }^{29}$. En effet, comment palier l'absence, la disparition d'un être, l'enfermement soudain et sans limites précises. Comment résister et avec quels mots? Les hommes ont opté, en général pour l'idéalisation de la figure aimée, la (re)création de l'amour et de la liberté qui se sont incarnés sous les traits de personnes-personnages mythico-mythologiques, éveillant en eux aussi bien le fantasme que la frustration extrême face à l'impalpable objet du désir.

Les femmes, quant à elles, ont choisi, consciemment ou inconsciemment, de représenter l'absent sous des traits reconnaissables et vraisemblables. Si l'idéalisation est parfois requise, elle l'est à une échelle moindre. Les compagnons désirés, disparus ou vivants, apparaissent en chaire et en os, comme dans ce poème de Delia Galará :

Je te sens tellement proche,

et je ne peux pas te toucher.

Les heures

semblent des siècles

et encore plus en cet après-midi

où la pluie nous accompagne,

\footnotetext{
que nos abrieran la puerta. Y si venía una requisa era desagradable. Nos gritaban sucias. Frente a eso, casi todas hemos dejado de menstruar en la cárcel. Fue como una neutralización de nuestra feminidad. ».

${ }^{27}$ Boisson nationale argentine.

${ }^{28}$ VV.AA $2006: 220$

${ }^{29}$ Il est absolument impossible de résumer dans un seul article ce qu'est la poésie carcérale féminine de la dernière dictature et ses différentes fonctions, c'est la raison pour laquelle il nous paraît judicieux d'insister sur l'aspect qui différencie de façon notoire l'écriture féminine et masculine.
} 
sa chanson...

se propage de façon monotone

dans l'air

et l'envie de te toucher

m'emplit la poitrine;

parcourir avec mes mains ton visage

les perdre sur ton corps...[... $]^{30}$

Ce poème sans titre fait preuve d'une certaine transparence et crédibilité dans son contenu. La personne décrite paraît exister au-delà du poème. D'ailleurs, si l'on ôte ce texte de son contexte, nous pourrions facilement penser qu'il s'agit d'une lettre poétique écrite à l'être aimé. Les genres littéraires se confondent alors pour créer une sensation de réel et de possible. Cela peut s'expliquer en partie par l'usage parcimonieux des adjectifs, contrairement aux hommes qui ont tendance à y avoir recours de façon abondante. La femme semble écrire à partir de souvenirs vécus, tandis que les hommes créent des situations nouvelles à partir d'images de leurs compagnes qu'ils ont magnifiées :

C'était à l'aube lorsque tu es entrée, / j'ai ouvert la fenêtre et senti ton arôme, / et je me suis mis à penser longuement / si toi et la liberté vous vous ressembliez. / Tu es le rêve auquel je rêve / et tu es le rêve que je vis / tu es la pensée sublime que je recherche, / la journée ensoleillée et la nuit éclairée par la lune. / Tu es la main qui guide ma main / qui crée et découvre. / En toi j'ai découvert l'harmonie / faite de musique, de lumière et de couleurs, / et l'instant transcendant / qui illumine nos vies / elle, c'est la possibilité / et toi, l'être / tu es, finalement / la beauté. ${ }^{31}$

Dans ce poème, composé dans la prison de La Plata par Rodolfo Novillo, l'admiration que le "je" poétique professe à son aimée dépasse la simple idéalisation pour atteindre le stade de la sublimation poétique -confirmée par le vers "tu es la pensée sublime que je recherche". Cependant, le choix du poète implique, précisément, une recherche sans certitude de rencontre. En associant la femme avec le "rêve" et la "pensée", il la met sur un piédestal impossible à atteindre, source d'un désir immense et insatiable. La personne décrite adopte ici des traits imprécis qui s'apparentent à un corps volatile, capable de diffuser un parfum envoûtant depuis les fenêtres de la cellule. De plus, il convient d'observer que la femme ressemble aux figures adorées dans les cultes religieux, ce qui peut s'observer au travers du champ lexical correspondant : "guide », « harmonie », «transcendant », « illumine ». La sacralisation de l'être aimé présente ici et dans de nombreux poèmes composés par des hommes est révélatrice d'un besoin et d'un manque quotidien. La poésie apparaît donc, tant pour les hommes que pour les femmes, comme un moyen de résister l'absence impossible à palier. Or, il est intéressant de constater que les femmes ont préféré représenter un amour concret et réel alors que

\footnotetext{
${ }^{30}$ Galará 2006 : 87. «Sans titre », prison de Villa Devoto, non daté. Nous traduisons. « Te siento tan cerca, / y no puedo tocarte. / Las horas / se me hacen siglos / y más en esta siesta / en que la lluvia nos acompaña, / su canción... / monótonamente se va extendiendo / en el aire / y las ganas de tenerte / me llenan el pecho; / recorrer con mis manos tu rostro / perderlas en tu cuerpo... [...]»

${ }^{31}$ Novillo $2003: 7$. « Tú y la belleza », prison de La Plata, 1979. Nous traduisons. « Fue al amanecer cuando tú entraste, / abrí la ventana y respiré tu aroma, / y estuve pensando largamente / si tú y la libertad se parecían. / Tú eres el sueño que sueño / y eres el sueño que vivo / eres el pensamiento sublime que busco, / el día de sol y la noche con luna. / Eres la mano que guía mi mano / que crea y descubre. / En ti descubrí la armonía / de música, luz y color, / y el instante trascendente / que ilumina nuestras vidas / ella, es la posibilidad / y tú, el ser / tú eres al fin / la belleza. ».
} 
les hommes ont opté pour la représentation fortement idéalisée de l'amour. Ce phénomène a été analysé par le poète et ancien prisonnier politique Carlos Liscano qui parle de « distance cosmique » entre le palpable et la réalité de la prison :

Nous parlions de ce que nous n'avions pas, des objets, des relations normales, de l'affection envers des êtres chers qui n'étaient pas avec nous et que, parfois, nous ne voyions pas depuis des années. Il y avait une distance cosmique entre la capacité presque infinie de la langue de nommer et le peu que nous pouvions vraiment nommer et montrer du doigt. ${ }^{32}$

Si le sacré implique, comme le signale Michel Butor, une idée de séparation ${ }^{33}$, la sacralisation empêche tout espoir d'atteindre l'objet du désir. À l'image de leur résistance quotidienne, concrète et organisée, les femmes paraissent avoir eu recours à la poésie afin d'extérioriser un manque qu'elles ont tout fait pour ne pas transformer en frustration insupportable. Ces actes de résistance carcérale, multiples et variés, sont l'illustration de ce que Pilar Calveiro nomme les «lignes de fugue », que les militaires ont tenté d'annuler et de stériliser à tout prix ${ }^{34}$. En effet et contre toute attente, les femmes ont produit des centaines de poèmes et des milliers de témoignages. À cet égard, la seule existence du livre collectif Nosotras, presas políticas (2006) démontre que la politique d'isolement géographique et de persécution physique et psychologique n'a pas pu contrer la volonté viscérale des femmes de résister.

\section{Conclusion}

Cette présentation succincte de ce qu'ont pu vivre les prisonnières politiques argentines est loin d'être complète mais peut permettre de comprendre un peu mieux la complexité de cette période. Et surtout, cela met en lumière, une fois de plus, l'incroyable capacité de l'être humain à revendiquer une dignité tant de fois bafouée. A l'instar des prisonnières du camp de concentration de Ravensbrück, qui ont-elles aussi trouvé la force de résister malgré l'oppression-l'opérette Le verfügbar aux enfers (1944-1945) de Germaine Tillion en est un exemple concret ${ }^{35}$ - les prisonnières politique argentines ont transformé des centres de détention-destruction en lieux de création. Ignorant les liens solidaires qui s'étaient tissés entre les femmes, qui allaient au-delà de l'appartenance politique de chacune, les autorités pénitentiaires et militaires ne purent pas atteindre les objectifs qu'elles s'étaient fixés ; ni en les classifiant, ni en les isolant, ni en les changeant constamment de cellules et de pavillons, ni en les exposant quotidiennement à des traitements inhumains.

\section{Bibliographie}

ANGENOT Marc, 2002, Interventions Critiques Vol I. Questions d'analyse du discours,

\footnotetext{
${ }^{32}$ Liscano 2007 : 242. Nous traduisons. « Hablábamos de lo que no teníamos, objetos, relaciones normales, afectos hacia seres queridos que no estaban con nosotros y a quienes a veces no veíamos desde hacía años. Había una distancia cósmica entre la casi infinita capacidad de la lengua de nombrar y lo poco que de verdad podíamos nombrar y señalar con el dedo. ».

${ }^{33}$ Butor 1995 : 16-17.

${ }^{34}$ Pilar Calveiro déclare dans son livre Poder y Desaparición que «l'objectif [des militaires] était d'obtenir l'information utile, mais aussi de faire craquer l'individu, de détruire le militant en annulant en lui toute ligne de fugue ou de résistance. ». Nous traduisons. « el objetivo era obtener información útil, pero además, quebrar al individuo, romper al militante anulando en él toda línea de fuga o resistencia. ». Calveiro $2006: 69$.

${ }^{35}$ Tillion 2007.
} 
de rhétorique et de théorie du discours social, Montréal, Nouvelle Série, Vol. VIII.

BUTOR Michel, 1995, L'utilité poétique, Saulxures, Circé.

CALVEIRO Pilar, 2006, Poder y desaparición, Los campos de concentración en Argentina. Buenos Aires, Colihue.

COMISIÓN PROVINCIAL DE LA MEMORIA, 2009, Detenidos Especiales, Presos Políticos, Fondo: Servicio Penitenciario Provincial, Coordination Ludmila da Silva Catela, Córdoba, Comisión provincial de la memoria/Archivo Provincial de la Memoria.

COMISIÓN Y ARCHIVO PROVINCIAL DE LA MEMORIA, mai 2010, Mujeres, silencios, dictadura. Diario de la memoria $\mathrm{N}^{\circ} 4$, año III, Córdoba.

GALARÁ Delia, 2006, Rehenes de nuestros sueños, Córdoba, Fojas Cero.

GARAÑO Santiago et PERTOT Werner, 2007, Detenidos-aparecidos, Presas y presos políticos desde Trelew a la dictadura, Buenos Aires, Biblos.

LISCANO Carlos, 2007, "Del caos a la literatura », Biblioteca Uruguaya de Psicoanálisis, Vol. VII-Literatura y Psicoanálisis, [consulté le 27/05/2012]. Disponible sur http://www.apuruguay.org/bup_pdf/bupVII-liscano.pdf

MOHADED Ana, 2010, El calzonazo, in red nosotras en el mundo, Córdoba, [consulté le 28/08/2014]. Disponible sur http://www.rednosotrasenelmundo.org/Se-vinoEl-calzonazo

NOVILLO Rodolfo, 2003, Los tesoros del Petizo, Córdoba, Éditions de l'auteur.

PLATÍA Marta, 19 août 2013, "Había una apropiación de las mujeres", in Página/12, Buenos Aires, [consulté le 06/05/2014]. Disponible sur http://www.pagina12.com.ar/diario/elpais/1-227095-2013-08-19.html

GARCÍA-ROMEU José, 2005, Dictature et littérature en Argentine: 1976-1983, Paris, L'Harmattan.

TILLION Germaine, 2007, Une opérette à Ravensbrück, Paris, Point.

VV.AA, 2006, Nosotras, presas políticas, Buenos Aires, Nuestra América. 